\title{
Impact of proton pump inhibitor treatment on gastrointestinal bleeding associated with non-steroidal anti-inflammatory drug use among post-myocardial infarction patients taking antithrombotics: nationwide study
}

\author{
Anne-Marie Schjerning Olsen, ${ }^{1,2}$ Jesper Lindhardsen, ${ }^{1}$ Gunnar H Gislason, ${ }^{1,3,4}$ Patricia McGettigan, ${ }^{2}$ \\ Mark A Hlatky, ${ }^{5}$ Emil Fosbøl, ${ }^{6}$ Lars Køber, ${ }^{6}$ Christian Torp-Pedersen, ${ }^{7}$ Morten Lamberts ${ }^{8}$
}

${ }^{1}$ Department of Cardiology,

Copenhagen University Hospital

Herlev and Gentofte, 2900 Hellerup, Denmark

${ }^{2}$ William Harvey Research Institute (WHRI) at Barts and the London School of Medicine and Dentistry, London, UK

${ }^{3}$ Danish Heart Foundation, Copenhagen, Denmark

${ }^{4}$ National Institute of Public Health, University of Southern

Denmark, Copenhagen, Denmark

${ }^{5}$ Stanford University School of Medicine, Stanford, CA, USA ${ }^{6}$ Department of Cardiology, Heart Centre, Copenhagen University Hospital Rigshospitalet,

Copenhagen, Denmark

${ }^{7}$ Department of Health, Science and Technology, Aalborg University, Aalborg, Denmark ${ }^{8}$ Department of Cardiology, Copenhagen University Hospital Herlev, Copenhagen, Denmark

Correspondence to:

A Schjerning Olsen

aols0073@geh.regionh.dk Additional material is published online only. To view please visit the journal online (http://dx.doi. org/10.1136/bmj.h5096)

Cite this as: $B M J$ 2015;351:h5096 doi: 10.1136/bmj.h5096

Accepted: 17 September 2015

\section{ABSTRACT}

\section{STUDY QUESTION}

What is the effect of proton pump inhibitors (PPIs) on the risk of gastrointestinal bleeding in post-myocardial infarction patients taking antithrombotics and treated with non-steroidal anti-inflammatory drugs (NSAIDs)?

METHODS

This was a nationwide cohort study based on linked administrative registry data from all hospitals in Denmark between 1997 and 2011. The study included patients aged 30 years and over admitted with a first myocardial infarction who survived at least 30 days after discharge. The association between PPIs and risk of gastrointestinal bleeding according to NSAID plus antithrombotic therapy was estimated using adjusted time dependent Cox regression models.

\section{STUDY ANSWER AND LIMITATIONS}

The use of PPIs was independently associated with decreased risk of gastrointestinal bleeding in postmyocardial infarction patients taking antithrombotics and treated with NSAIDs. Of 82955 post-myocardial infarction patients (mean age 67.4 years, 64\% $(n=53070)$ men), all of whom were taking single or dual antithrombotic therapy, $42.5 \%(n=35233)$ filled at least one prescription for NSAIDs and $45.5 \%(n=37771)$ received PPIs. Over a mean follow-up of 5.1 years, 3229 gastrointestinal bleeds occurred. The crude incidence rates of bleeding (events/100 person years) on NSAID plus antithrombotic therapy were 1.8 for patients

\section{WHAT IS ALREADY KNOWN ON THIS TOPIC}

Although administration of non-steroidal anti-inflammatory drugs (NSAIDs) to post-myocardial infarction patients is discouraged owing to their cardiovascular risks, NSAIDs are still widely used because painful conditions are common

Among post-myocardial infarction patients, bleeding complications have been associated with both antithrombotic and NSAID treatment

The effect of proton pump inhibitors (PPIs) on the risk of gastrointestinal bleeding in post-myocardial infarction patients taking antithrombotics who are treated with NSAIDs is unknown

\section{WHAT THIS STUDY ADDS}

Concurrent use of PPIs was independently associated with decreased risk of gastrointestinal bleeding in post-myocardial infarction patients taking antithrombotics who were treated with NSAIDs

This finding was independent of antithrombotic treatment regimen, type of NSAID, and type of PPI used

Post-myocardial infarction patients in whom NSAIDs are judged necessary might benefit from PPI treatment as well

taking PPIs and 2.1 for those not taking PPIs. The adjusted risk of bleeding was lower with PPI use (hazard ratio $0.72,95 \%$ confidence interval 0.54 to 0.95 ) regardless of antithrombotic treatment regimen, type of NSAID, and type of PPI used. The main limitation of the study is its observational nonrandomised design. The results suggest that PPI treatment probably has a beneficial effect regardless of underlying gastrointestinal risk and that when NSAIDs cannot be avoided in post-myocardial infarction patients, physicians might prescribe a PPI as well. The study does not clarify whether PPIs might be safely omitted in specific subgroups of patients with a low risk of gastrointestinal bleeding.

\section{WHAT THIS STUDY ADDS}

In post-myocardial infarction patients, bleeding complications have been associated with both antithrombotic and NSAID treatment. Concurrent use of PPIs was independently associated with a decreased risk of gastrointestinal bleeding in postmyocardial infarction patients taking antithrombotics and NSAID, regardless of antithrombotic treatment regimen, type of NSAID, and type of PPI used.

FUNDING, COMPETING INTERESTS, DATA SHARING AMSO has received a grant from the Danish Council of Independent Research (grant 12-132760). GHG is supported by an unrestricted research scholarship from the Novo Nordisk Foundation.

\section{Introduction}

Antithrombotic treatments are widely used in postmyocardial infarction patients to reduce thromboembolic risk, but at the cost of increased risk of bleeding. ${ }^{12}$ Gastrointestinal haemorrhage is the most common serious bleeding complication associated with antithrombotic treatment. ${ }^{1}$ Non-steroidal anti-inflammatory drugs (NSAIDs) are effective and widely used analgesics. Much attention has been given to their gastrointestinal safety..$^{3-7}$ They have been shown to carry a substantial independent risk of bleeding in cardiovascular patients when co-prescribed with antithrombotics, even with short term use ( $<14$ days).$^{8-10}$ Randomised controlled trials have reported that proton pump inhibitors (PPIs) reduce rates of recurrent gastrointestinal bleeding in patients receiving antithrombotics. ${ }^{112}$ Although NSAIDs are discouraged in post-myocardial infarction patients, pain is a common condition and, in practice, they are frequently prescribed. ${ }^{1314}$ American specialist cardiology and gastroenterology guidelines recommend that 
PPIs should be used with both antithrombotic and NSAID treatments in patients judged to be at high risk of gastrointestinal bleeding. ${ }^{15}$ In the United Kingdom, the National Institute for Health and Care Excellence (NICE) recommends routine PPI co-prescription with NSAIDs for everyone aged 45 years and older with osteoarthritis, rheumatoid arthritis, or chronic low back pain, ${ }^{16}$ as well as for people taking antithrombotics who are at high risk of gastrointestinal adverse effects including bleeding. ${ }^{17}$ However, the potential benefit of PPI therapy used with antithrombotic/ NSAID combinations in post-myocardial infarction patients irrespective of gastrointestinal risk is unknown. We therefore investigated the effect of PPIs on the risk of bleeding in Danish post-myocardial infarction patients taking antithrombotics who were also treated with NSAIDs.

\section{Methods}

\section{Data sources}

Diagnostic data came from the Danish National Patient Registry, which using ICD-10 (international classification of diseases, 10th revision) to classify hospital admissions (supplementary table 1). ${ }^{18}$ Each hospital admission is registered with one main discharge diagnosis and one or more supplementary diagnoses if appropriate. Information on vital status (dead or alive) came from the civil registration system through Statistics Denmark. We obtained primary, secondary, and contributing causes of death recorded by a physician from the National Causes of Death Registry. The National Prescription Registry provided information on the date of dispensing, quantity dispensed, strength, and formulation of all drugs dispensed from Danish pharmacies and classified according to the Anatomical Therapeutic Chemical (ATC) system (supplementary table 2). The partial reimbursement of drug expenses by the Danish healthcare system requires all pharmacies to register each drug dispensed in the National Prescription Registry, ensuring complete registration. ${ }^{19}$ In Denmark, every resident has a permanent unique civil registration number that enables linkage across administrative registries.

\section{Study population and follow-up}

We identified all patients aged 30 years and over in the National Patient Registry between 1997 and 2011 who had a primary diagnosis of acute myocardial infarction (ICD-10 code I21 to I22), received antithrombotics, and survived at least 30 days from discharge (date of inclusion). The 30 day restriction defined a quarantine period. The follow-up period started after the 30 day quarantine period to minimise risk of immortal time bias. To avoid selection bias, we used a new user design, excluding patients who collected a prescription for an NSAID during the quarantine period $(n=5711)$. Patients were followed until one of the following events (whichever came first): event of interest, emigration, death, or end of study period (31 December 2011). The diagnosis of myocardial infarction has been validated with a specificity exceeding $90 \% .^{20}$

\section{Antithrombotic, PPI, and NSAID treatment}

We used claimed prescriptions of aspirin or clopidogrel to characterise patients as receiving either antithrombotic monotherapy (aspirin or clopidogrel) or dual therapy. We excluded post-myocardial infarction patients who received no antithrombotic $(n=15353)$ or an oral anticoagulant only ( $\mathrm{n}=1440)$. We identified all claimed prescriptions for NSAIDs (ATC M01A, excluding glucosamine (M01AX05)). We categorised rofecoxib and celecoxib as cyclo-oxygenase-2 selective (COX 2) inhibitors; ibuprofen, diclofenac, and naproxen as non-selective NSAIDs; and all other NSAIDs as "other" NSAIDs. The only NSAID available in Denmark without prescription is ibuprofen (since 2001). We categorised PPI treatment in one group (ATC A02BC); we also examined five individual PPIs-omeprazole, lansoprazole, pantoprazole, esmoprazole, and rabeprazole.

We calculated exposure periods for NSAIDs and PPIs for each patient by estimating a daily dose after comparing the accumulated dose and the elapsed time from consecutive prescriptions for the drug under investigation. We determined ongoing exposure by dividing the number of tablets/capsules dispensed by the estimated average dosage. If only one prescription was registered for an individual, we used a standard dosage, defined as the minimal recommended dosage, to estimate the daily dose. We used information on increasing or decreasing dosage only to continuously assess whether tablets were available. We defined exposure as having occurred when patients had drug available and discontinuation as when they had no more drug available. Methods for determining dose and treatment duration have been described previously. ${ }^{2122}$ For most patients, treatment regimens changed during the study period, so we treated NSAID and PPI use in the analysis as time varying exposures-that is, patients changed exposure group according to claimed prescriptions. Each patient's exposure group at inclusion defined baseline treatment, shown in the table with the covariante distributions (supplementary figure).

\section{Comorbidity}

We identified comorbidities from previous diagnoses and at discharge from the index myocardial infarction, as specified in the Ontario acute myocardial infarction mortality prediction rule, and potential risk factors for bleeding (previous bleeding, alcohol consumption, liver disease, and ulcers). ${ }^{23}$ The Ontario acute myocardial infarction mortality prediction rule is a logistic regression model that predicts 30 day and one year mortality by using 11 variables determinable from hospital discharge databases (age, sex, shock, diabetes with complications, congestive heart failure, cancer, cerebrovascular disease, pulmonary oedema, acute renal failure, chronic renal failure, cardiac dysrhythmia). In our analyses, we incorporated each variable as a covariate and permitted diagnoses up to one year previously (supplementary table 1). ${ }^{24}$ To account for accumulation of risk factors during follow-up, we did an analysis including all variables in the main analysis as time dependent (exempt for inclusion year and 
percutaneous coronary intervention status (considered as related to myocardial infarction inclusion criteria) and previous peptic ulcer disease (considered an intermediate variable of the primary outcome during follow-up)). During the entire span of the database, patients could change status on the date of exposure.

\section{Outcome}

We defined the primary outcome of gastrointestinal bleeding as hospital admission for or death from a bleeding gastrointestinal ulcer, haematemesis, melena, or unspecified gastrointestinal bleeding from the National Causes of Death Register and National Patient Register. ${ }^{25}$ Occurrence and type of bleeding as recorded in hospital databases have shown a positive predictive value of $89-99 \%$.

\section{Statistical methods}

We calculated crude incidence rates as number of events per 100 person years according to the different treatment regimens. We estimated the effects of PPI treatment on gastrointestinal bleeding with adjusted Cox proportional hazards models in terms of hazard ratios and 95\% confidence intervals for gastrointestinal bleeding with the drug exposure continuously updating-that is, as time varying exposure allocated according to treatment regimen. We considered patients to be at risk only when exposed to the drug (during active treatment). Each patient could have multiple treatment groups throughout follow-up. We calculated risk time (person years) only for the active treatment period. The timescale in the Cox model was days passed since inclusion. We adjusted all models for age, sex, year of index hospital admission, concomitant drugs, comorbidity, and percutaneous coronary intervention status. We did additional analyses to assess any association between PPI use and individual antithrombotic regimens and NSAIDs.

We did nine sensitivity analyses. (1) To take account of any effect of over the counter NSAID use, we ended follow-up at 2001. (2) To take account of dabigatran or ticagrelor use (released in Denmark, August 2011), we

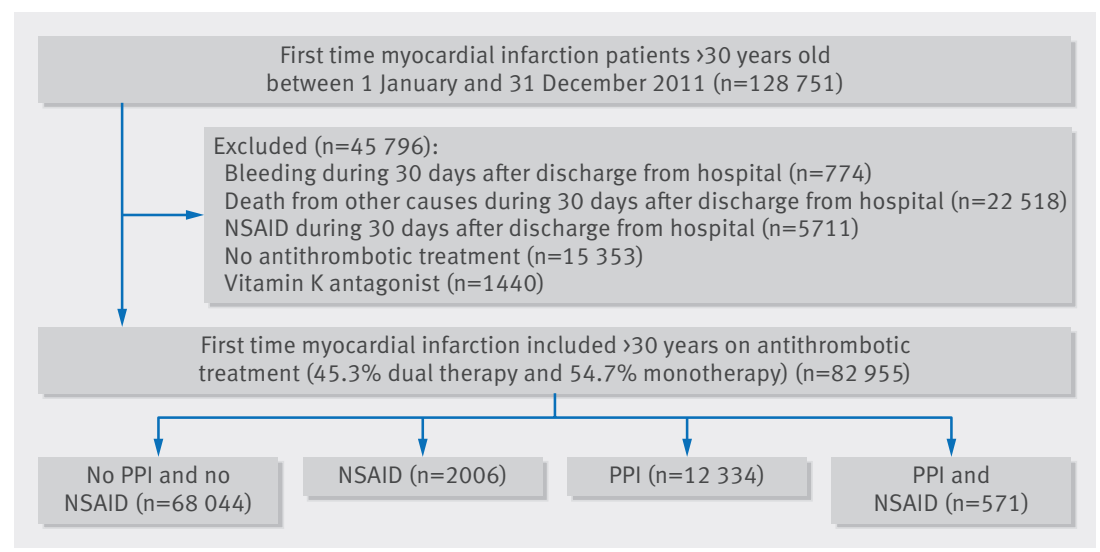

Fig 1 | Flow chart of study population. Quarantine period=30 days after discharge from hospital. Cohort consisted of patients who were alive after quarantine period and who were taking single or dual antithrombotic treatment. Cohort was further divided into four groups according to non-steroidal anti-inflammatory drug (NSAID) and proton pump inhibitors (PPI) use ended follow-up in December 2010. (3) We examined cardiovascular death as a solo endpoint. (4) We stratified the cohort at 65 years to take account of guidelines recommending PPIs for people over 65 taking antithrombotic treatment. (5) We controlled for the variables included in the HAS-BLED score. (6) We did an analysis including all covariates as time dependent. (7) We stratified the population in two groups: high (previous bleeding) and low (no previous bleeding) risk of gastrointestinal bleeding. (8) Although the indication for NSAID use was not systematically available, we were able to do an analysis of patients with rheumatoid arthritis. (9) We examined the effect of duration of NSAID treatment (0-14 days and $>14$ days).

We confirmed the validity of the proportional hazard assumption, linearity of continuous variables, and lack of interaction and chose a significance level of 0.05. We used SAS 9.2 and Stata 11.0 for all statistical calculations.

\section{Patient involvement}

No patients were involved in setting the research question or the outcome measures; nor were they involved in the design and implementation of the study. There are no plans to involve patients in dissemination.

\section{Results}

A total of 128751 patients were admitted with a first myocardial infarction in the period 1997 to 2011, of whom $82955(64.4 \%)$ were taking single or dual antithrombotic therapy and met the study inclusion criteria (fig 1). Baseline characteristics included 67.4 years mean age, 64\% male, and $6.6 \%$ with a history of previous gastrointestinal bleeding (table). A total of 35233 (42.5\%) patients had one or more NSAID prescription claim after discharge; 37711 (45.5\%) received a PPI, and 10613 (12.8\%) had concurrent NSAID and PPI exposure (fig 2). The proportions receiving a PPI in the year after their first myocardial infarction increased from 15\% in 1997 to 37\% by 2010 . The proportions receiving NSAIDs peaked at 20\% in 2002 and subsequently declined, whereas the proportions receiving a PPI concurrently with an NSAID increased from less than $10 \%$ to more than $30 \%$ by 2010 (fig 2 ).

\section{Gastrointestinal bleeding}

We identified 3229 gastrointestinal bleeding events, 282 $(8.7 \%)$ of which were fatal. A total of 327 events were registered during NSAID treatment. The overall crude incidence rate was 0.8 (95\% confidence interval 0.7 to 0.8 ) events per 100 person years The crude incidence rate of gastrointestinal bleeding on NSAID treatment was 2.1 (1.8 to 2.4) events per 100 person years without concurrent PPI treatment and 1.8 (1.4 to 2.4) with concurrent PPI treatment (fig 3 ). After multivariable adjustment for baseline differences, use of PPIs concurrently with combined antithrombotic and NSAID treatment was associated with a significantly lower risk of gastrointestinal bleeding (hazard ratio 0.72, 95\% confidence interval 0.54 to 0.95 ) compared with treatment without concurrent PPIs (fig 3). Compared with combined antithrombotic and NSAID treatment (without concurrent 


\begin{tabular}{|c|c|c|c|c|c|}
\hline \multicolumn{6}{|c|}{$\begin{array}{l}\text { Baseline characteristics of total study population and individual antithrombotic treatment groups. Values are numbers (percentages) unless stated } \\
\text { otherwise }\end{array}$} \\
\hline Characteristic & $\begin{array}{l}\text { Total population } \\
(\mathrm{n}=82 \text { 955) }\end{array}$ & $\begin{array}{l}\text { No PPI and no } \\
\text { NSAID }(n=68044)\end{array}$ & NSAID $(n=2006)$ & $\operatorname{PPI}(n=12334)$ & $\begin{array}{l}\text { PPI and NSAID } \\
(\mathrm{n}=571)\end{array}$ \\
\hline Mean (SD) age, years & $67.4(13.3)$ & $66.7(13.3)$ & $67.7(12.8)$ & $71.4(12.8)$ & $68.7(13.0)$ \\
\hline Male sex & $53070(64.0)$ & $44802(65.8)$ & $1199(59.8)$ & $6772(54.9)$ & $297(52.0)$ \\
\hline \multicolumn{6}{|l|}{ Comorbidities: } \\
\hline Cardiac arrhythmias & $7624(9.2)$ & $5821(8.6)$ & $164(8.2)$ & 1590 (12.9) & $49(8.6)$ \\
\hline Peripheral vascular disease & $3132(3.8)$ & $2264(3.3)$ & $69(3.4)$ & $776(6.3)$ & $23(4.0)$ \\
\hline Cerebral vascular disease & $3716(4.5)$ & $2748(4.0)$ & $64(3.2)$ & $878(7.1)$ & $26(4.6)$ \\
\hline Diabetes with complications & 3404 (4.1) & $2498(3.7)$ & $89(4.4)$ & $789(6.4)$ & $28(4.9)$ \\
\hline Acute renal failure & $628(0.8)$ & $339(0.5)$ & $10(0.5)$ & $268(2.2)$ & $11(1.9)$ \\
\hline Chronic renal failure & $1027(1.2)$ & $603(0.9)$ & $12(0.6)$ & $402(3.3)$ & $10(1.8)$ \\
\hline Malignancy & $1796(2.2)$ & $1296(1.9)$ & $35(1.7)$ & $442(3.6)$ & $23(4.0)$ \\
\hline Shock & $216(0.3)$ & $143(0.2)$ & $1(0.1)$ & $70(0.6)$ & $2(0.4)$ \\
\hline Chronic obstructive pulmonary disease & $690(0.8)$ & $522(0.8)$ & $12(0.6)$ & $148(1.2)$ & $8(1.4)$ \\
\hline Previous bleeding & $5511(6.6)$ & $3466(5.1)$ & $115(5.7)$ & $1826(14.8)$ & $66(11.6)$ \\
\hline Liver disease & $954(1.2)$ & $657(1.0)$ & $22(1.1)$ & $267(2.2)$ & $8(1.4)$ \\
\hline Peptic ulcer & $3481(4.2)$ & $1706(2.5)$ & $44(2.2)$ & $1671(13.6)$ & $60(10.5)$ \\
\hline Alcohol & $3255(3.9)$ & $2415(3.6)$ & $83(4.1)$ & $714(5.8)$ & $43(7.5)$ \\
\hline Percutaneous coronary intervention & $32376(39.0)$ & $26764(39.3)$ & $691(34.5)$ & $4725(38.3)$ & $196(34.3)$ \\
\hline \multicolumn{6}{|l|}{ Concomitant drugs: } \\
\hline$\beta$ blockers & 60789 (73.3) & $50541(74.3)$ & $1405(70.0)$ & $8446(68.5)$ & $394(69.0)$ \\
\hline Angiotensin converting enzyme inhibitors & $31748(38.3)$ & $25821(38.0)$ & $769(38.3)$ & $4928(40.0)$ & $230(40.3)$ \\
\hline Statins & $48829(58.9)$ & $40214(59.1)$ & $1156(57.6)$ & $7107(57.6)$ & $352(61.7)$ \\
\hline Spironolactone & $4771(5.8)$ & $3560(5.2)$ & $116(5.8)$ & $1048(8.5)$ & $47(8.2)$ \\
\hline Loop diuretics & $22020(26.5)$ & $16685(24.5)$ & $572(28.5)$ & $4563(37.0)$ & $200(35.0)$ \\
\hline Glucose lowering drugs & $6388(7.7)$ & $4860(7.1)$ & $195(9.7)$ & $1263(10.2)$ & $70(12.3)$ \\
\hline
\end{tabular}

PPI), taking antithrombotics only (without NSAID or PPI) or antithrombotics with concurrent PPI treatment (without NSAID) were each associated with a lower risk of gastrointestinal bleeding. The bleeding risk associated with concurrent PPI and NSAID treatment was similar for each antithrombotic regimen (supplementary table 3). PPIs taken concurrently with NSAID and dual antithrombotic therapy were associated with a reduced

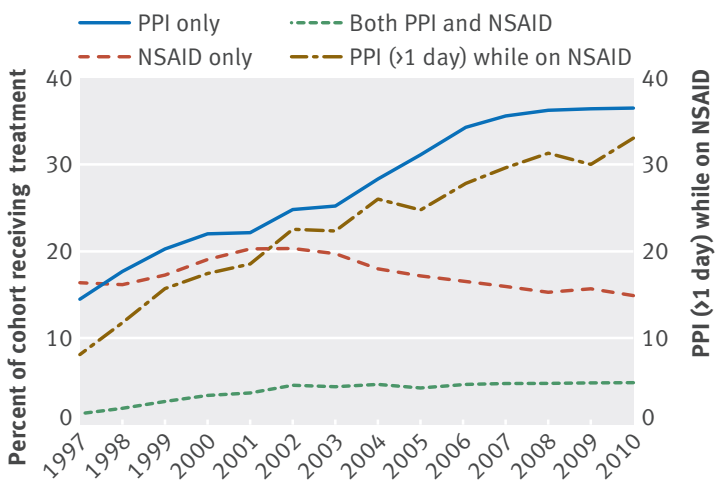

Year

Fig 2 Distribution of use of proton pump inhibitor (PPI), non-steroidal anti-inflammatory drug (NSAID), and both one year after myocardial infarction among patients taking single or dual antithrombotic treatment. Left side $Y$ axis: curves represent percentages of entire cohort receiving PPI only, NSAID only, and both PPI and NSAID within one year from study inclusion after myocardial infarction. Right side Y axis: curve represents percentage receiving PPI (for at least one day) while on NSAID treatment within one year from study inclusion after myocardial infarction risk of gastrointestinal bleeding (incidence rate 2.5 (1.3 to 4.8) events per 100 person years) compared with NSAID and dual antithrombotic therapy without concurrent PPIs (5.2 (3.9 to 6.8) events per 100 person years) (hazard ratio $0.41,0.20$ to 0.84 ).

Different NSAID groups (selective COX 2 inhibitors, non-selective NSAIDs, other NSAIDs) and PPIs were associated with similar adjusted hazard ratios and crude incidence rates as for overall NSAID treatment (fig 3, top). Individual PPIs (omeprazole, pantoprazole, lanzoprazole, and esmoprazole) were each associated with reduced bleeding risks (fig 3, bottom). For individual NSAIDs (rofecoxib, celecoxib, diclofenac, ibuprofen, naproxen), hazard ratios for bleeding were lower with concurrent PPI use than without, but not significantly so owing to small numbers of events individually (supplementary table 4).

\section{Sensitivity analyses}

None of the sensitivity analyses changed the results appreciably (web appendix).

\section{Discussion}

This nationwide study suggests that PPIs reduce the risk of gastrointestinal bleeding associated with NSAID use among post-myocardial infarction patients taking antithrombotics. Four PPIs (omeprazole, pantoprazole, lanzoprazole, esmoprazole) were individually associated with a lower risk of bleeding compared with nonuse for the antithrombotic regimens examined and irrespective of whether selective COX 2 inhibitors or non-selective NSAIDs were prescribed. 


\begin{tabular}{|c|c|c|c|c|}
\hline Treatment & No & $\begin{array}{c}\text { Crude rates } \\
\text { (per } 100 \text { person years) }\end{array}$ & $\begin{array}{l}\text { Hazard ratio } \\
(95 \% \mathrm{CI})\end{array}$ & $\begin{array}{l}\text { Hazard ratio } \\
(95 \% \mathrm{Cl})\end{array}$ \\
\hline NSAID without PPI & 267 & 2.1 (1.8 to 2.4$)$ & 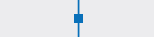 & 1.0 (reference) \\
\hline NSAID + PPI & 60 & 1.8 (1.4 to 2.4$)$ & $\rightarrow-$ & 0.72 (0.54 to 0.95$)$ \\
\hline \multicolumn{5}{|l|}{ Types of NSAIDs } \\
\hline COX 2 inhibitor $+\mathrm{PPI}$ & 7 & 2.1 (1.0 to 4.4$)$ & $\rightarrow-1$ & $0.61(0.29$ to 1.29$)$ \\
\hline Non-selective NSAID + PPI & 39 & 1.8 (1.3 to 2.5$)$ & & $0.76(0.54$ to 1.06$)$ \\
\hline Other NSAID + PPI & 14 & $1.8(1.1$ to 3.1$)$ & $\rightarrow-$ & $0.67(0.39$ to 1.14$)$ \\
\hline NSAID without PPI & 267 & 2.1 (1.8 to 2.4$)$ & 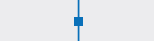 & 1.0 (reference) \\
\hline NSAID + PPI & 60 & $1.8(1.4$ to 2.4$)$ & $\rightarrow$ & $0.72(0.54$ to 0.95$)$ \\
\hline \multicolumn{5}{|l|}{ Types of PPIs } \\
\hline NSAID + omeprazol & 10 & 1.3 (0.7 to 2.5$)$ & & $0.55(0.29$ to 1.04$)$ \\
\hline NSAID + pantoprazol & 19 & 2.3 (1.5 to 3.7$)$ & & $0.84(0.53$ to 1.35$)$ \\
\hline NSAID + lanzoprazol & 16 & 1.8 (1.1 to 3.0$)$ & 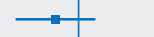 & $0.74(0.45$ to 1.23$)$ \\
\hline NSAID + esmoprazol & 14 & $1.8(1.1$ to 3.1$)$ & $\longrightarrow$ & $0.68(0.40$ to 1.16$)$ \\
\hline
\end{tabular}

Fig 3 | Cox proportional hazard analysis risk of gastrointestinal bleeding on antithrombotic treatment with and without non-steroidal anti-inflammatory drug (NSAID) and proton pump inhibitor (PPI) treatment in patients with previous myocardial infarction (MI). Reference is antithrombotic treatment plus concomitant NSAID without PPI treatment (vertical line indicates reference level)
Adverse cardiovascular effects associated with NSAIDs have led to discouragement of their use in patients with cardiovascular disease. ${ }^{2627}$ NSAIDs have also been associated with a substantial independent risk of bleeding in patients already taking antithrombotics. ${ }^{89}$ Nevertheless, they continue to be prescribed quite extensively for patients with cardiovascular risks. Previous studies have reported that around $40 \%$ of Danish patients with myocardial infarction are exposed to NSAIDs, ${ }^{131422}$ consistent with the $42.5 \%$ found here. PPIs reduce the risks of gastrointestinal complications associated with antithrombotics and with NSAIDs, including among patients at high risk treated with selective or non-selective NSAIDs. ${ }^{28-32}$ Our results support these findings in showing that concurrent PPIs were associated with a reduced risk of gastrointestinal bleeding among post-myocardial infarction patients taking both NSAIDs and antithrombotics.

Concern has been expressed that some PPIs might diminish the antiplatelet effect of clopidogrel, most likely through inhibition of CYP2C19 mediated conversion of clopidogrel into its active form, but studies have been contradictory and definitive data are awaited. ${ }^{33}$ A similar situation exists regarding concerns that PPIs might impair the cardiovascular protective efficacy of aspirin. ${ }^{12}$ Consequently, PPIs are recommended for aspirin treated patients at high risk of gastrointestinal bleeding. American specialty guidelines recommend that in patients at high risk of gastrointestinal bleeding, a PPI should be used concomitantly with antithrombotics and with NSAID treatment. ${ }^{15}$. Our data suggest that PPI treatment probably has a beneficial effect regardless of underlying gastrointestinal risk and that when NSAIDs cannot be avoided in post-myocardial infarction patients, physicians might prescribe a PPI as well. Our study does not clarify whether PPIs might be safely omitted in specific subgroups of patients with a low risk of gastrointestinal bleeding.

\section{Limitations of study}

The main limitation of this study is its observational non-randomised design. Furthermore, we do not have information on whether patients stop taking antithrombotics when treated with NSAIDs; however, given post-myocardial infarction treatment guidelines, we think that this is unlikely. We cannot exclude a possible effect of unmeasured confounders. However, if an unmeasured confounder or a combination of confounders were present in $20 \%$ of the NSAID treated cohort, our calculations suggest that the confounder would have to increase the risk by a factor of 1.8 to 3.5 to explain the increased risk observed. Existence of such a confounder or combination of confounders is unlikely, but not impossible, as we had no information on other risk factors such as smoking, lipid concentrations, or body mass index. Moreover, we did an analysis with continuous assessment of co-variables to account for accumulation of risk factors during follow-up to further minimise the effect of potential confounders; the results were unaffected. We did not have information about the indication for NSAID treatment, but this was probably for non-cardiac disease, as NSAIDs are not used to treat ischaemic heart disease. Having users treated with the drug(s) under investigation before inclusion could result in confounding (healthy user effect). Our estimates when we excluded prevalent NSAID users remained the same, so it is unlikely that confounding by indication alone could drive the observed results. The only NSAID available over the counter in Denmark is ibuprofen (since 2001) in low doses $(200 \mathrm{mg})$ and in limited quantity. Restricting the analyses to $1997-2000$ did not change our results. Aspirin is also available without prescription, but because of partial reimbursement of drug expenses, chronic users, in particular those with a history of myocardial infarction, are most likely to be issued with a prescription for aspirin as thrombo-prophylaxis. ${ }^{3435}$ For these reasons, we think unrecorded over the counter drug use is unlikely to have had a major effect on the study results. Several estimates of a beneficial effect of concomitant specific PPI and specific NSAID treatment on risk of gastrointestinal bleeding were non-significant. This was potentially owing to a low number of events. Nevertheless, all estimates were comparable to our main analysis.

\section{Conclusion}

This study of a real life cohort of post-myocardial infarction patients taking antithrombotics suggests that use of a PPI diminishes the risk of gastrointestinal bleeding associated with NSAID treatment, regardless of the type of NSAID or PPI prescribed. Consequently, when NSAIDs cannot be avoided in post-myocardial infarction patients, physicians might consider prescribing a PPI as well.

Contributors: AMSO and ML made primary contributions to study design, data collection, data analysis, interpretation of results, and writing the manuscript. ML helped to write the first draft. JL, GHG, and CT-P contributed to the study design. All authors contributed to the interpretation of results and critical revision of the manuscript; all approved the final manuscript. AMSO is the guarantor. 
Funding: AMSO has received a grant from the Danish Council of Independent Research (grant 12-132760). GHG is supported by an unrestricted research scholarship from the Novo Nordisk Foundation. The funding sources had no influence on study design, interpretation of the results, or the decision to submit the article.

Competing interests: All authors have completed the ICMJE uniform disclosure form at www.icmje.org/coi_disclosure.pdf (available on request from the corresponding author) and declare: no support from any organisation for the submitted work; no financial relationships with any organisations that might have an interest in the submitted work in the previous three years, no other relationships or activities that could appear to have influenced the submitted work.

Ethical approval: The Danish Data Protection Agency approved this study (No 2007-58-0015; internal ref: GEH-2014-014 I-Suite 02732), and data at the individual level were made available to us such that specific patients could not be identified. Retrospective register studies do not by law require ethical approval in Denmark.

Transparency declaration: The lead author (the manuscript's guarantor) affirms that this manuscript is an honest, accurate, and transparent account of the study being reported; that no important aspects of the study have been omitted; and that any discrepancies from the study as planned (and, if relevant, registered) have been explained.

Data sharing: No additional data available.

This is an Open Access article distributed in accordance with the Creative Commons Attribution Non Commercial (CC BY-NC 4.0) license, which permits others to distribute, remix, adapt, build upon this work non-commercially, and license their derivative works on different terms, provided the original work is properly cited and the use is non-commercial. See: http://creativecommons.org/licenses/ by-nc/4.0/.

1 Sorensen R, Hansen ML, Abildstrom SZ, et al. Risk of bleeding in patients with acute myocardial infarction treated with different combinations of aspirin, clopidogrel, and vitamin K antagonists in Denmark: a retrospective analysis of nationwide registry data. Lancet 2009;374:1967-74.

2 Hart RG, Pearce LA, Aguilar MI. Meta-analysis: antithrombotic therapy to prevent stroke in patients who have nonvalvular atrial fibrillation. Ann Intern Med 2007;146:857-67.

3 Bhala N Emberson! Merhi A et al Vascular and upper gastrointestinal effects of non-steroidal anti-inflammatory drugs: meta-analyses of individual participant data from randomised trials. Lancet 2013;382:769-79.

4 McGettigan P. Henry D. Cardiovascular risk with non-steroidal anti-inflammatory drugs: systematic review of population-based controlled observational studies. PLoS Med 2011;8:e1001098.

5 Trelle S, Reichenbach S, Wandel S, et al. Cardiovascular safety of non-steroidal anti-inflammatory drugs: network meta-analysis. BMJ 2011;342:C7086.

6 Bombardier C, Laine L, Reicin A, et al. Comparison of upper gastrointestinal toxicity of rofecoxib and naproxen in patients with rheumatoid arthritis. N Engl/ Med 2000;343:1520-8, 2 p following 8.

7 Hernandez-Diaz S, Varas-Lorenzo C, Garcia Rodriguez LA. Non-steroidal antiinflammatory drugs and the risk of acute myocardial infarction. Basic Clin Pharmacol Toxicol 2006;98:266-74

8 Davidson BL, Verheijen S, Lensing AW, et al. Bleeding risk of patients with acute venous thromboembolism taking nonsteroidal anti-inflammatory drugs or aspirin. JAMA Intern Med 2014; $174: 947-53$.

9 Lamberts M, Lip GY, Hansen ML, et al. Relation of nonsteroidal anti-inflammatory drugs to serious bleeding and thromboembolism risk in patients with atrial fibrillation receiving antithrombotic therapy: a nationwide cohort study. Ann Intern Med 2014;161:690-8.

10 Schjerning Olsen AM, Gislason GH, McGettigan P, et al. Association of NSAID use with risk of bleeding and cardiovascular events in patients receiving antithrombotic therapy after myocardial infarction. JAMA 2015;313:805-14

11 Bhatt DL, Cryer BL, Contant CF, et al. Clopidogrel with or without omeprazole in coronary artery disease. N Engl J Med 2010;363:1909-17.

12 Agewall S, Cattaneo M, Collet JP, et al. Expert position paper on the use of proton pump inhibitors in patients with cardiovascular disease and antithrombotic therapy. Eur Heart J 2013;34:1708-13, 13a-b.

13 Schjerning Olsen AM, Fosbol EL, Lindhardsen I, et al. Duration of treatment with nonsteroidal anti-inflammatory drugs and impact on risk of death and recurrent myocardial infarction in patients with prior myocardial infarction: a nationwide cohort study. Circulation 2011:123:2226-35.

14 Olsen AM, Fosbol EL, Lindhardsen J, et al. Long-term cardiovascular risk of nonsteroidal anti-inflammatory drug use according to time passed after first-time myocardial infarction: a nationwide cohort study. Circulation 2012;126:1955-63.
15 Abraham NS, Hlatky MA, Antman EM, et al. ACCF/ACG/AHA 2010 expert consensus document on the concomitant use of proton pump inhibitors and thienopyridines: a focused update of the ACCF/ACG/ AHA 2008 expert consensus document on reducing the gastrointestinal risks of antiplatelet therapy and NSAID use. A report of the American College of Cardiology Foundation Task Force on Expert Consensus Documents. J Am Coll Cardiol 2010;56:2051-66.

16 National Institute for Health and Care Excellence. NSAIDs - prescribing issues. 2015. cks.nice.org.uk/nsaids-prescribing-issues\#!scenario.

17 National Institute for Health and Care Excellence. Antiplatelet treatment. 2015. cks.nice.org.uk/antiplatelet-treatment\#!scenario1.

18 Andersen TF, Madsen M, Jorgensen J, Mellemkjoer L, Olsen JH. The Danish National Hospital Register: a valuable source of data for modern health sciences. Dan Med Bull 1999:46:263-8.

19 Gaist D, Sorensen HT, Hallas J. The Danish prescription registries. Dan Med Bull 1997:44:445-8.

20 Madsen M, Davidsen M, Rasmussen S, Abildstrom SZ, Osler M. The validity of the diagnosis of acute myocardial infarction in routine statistics: a comparison of mortality and hospital discharge data with the Danish MONICA registry. J Clin Epidemiol 2003:56:124-30.

21 Fosbol EL, Gislason GH, Jacobsen S, et al. The pattern of use of non-steroidal anti-inflammatory drugs (NSAIDs) from 1997 to 2005: a nationwide study on 4.6 million people. Pharmacoepidemiol Drug Saf 2008:17:822-33

22 Gislason GH, Jacobsen S, Rasmussen JN, et al. Risk of death or reinfarction associated with the use of selective cyclooxygenase-2 inhibitors and nonselective nonsteroidal antiinflammatory drugs after acute myocardial infarction. Circulation 2006;113:2906-13.

23 Tu JV, Austin PC, Walld R, Roos L, Agras J, McDonald KM. Development and validation of the Ontario acute myocardial infarction mortality prediction rules. I Am Coll Cardiol 2001:37:992-7.

24 Rasmussen S, Zwisler AD, Abildstrom SZ, Madsen JK, Madsen M. Hospital variation in mortality after first acute myocardial infarction in Denmark from 1995 to 2002: lower short-term and 1-year mortality in high-volume and specialized hospitals. Med Care 2005;43:970-8.

25 Lamberts M, Olesen JB, Ruwald MH, et al. Bleeding after initiation of multiple antithrombotic drugs, including triple therapy, in atrial fibrillation patients following myocardial infarction and coronary intervention: a nationwide cohort study. Circulation 2012;126:1185-93.

26 Antman EM, Bennett JS, Daugherty A, Furberg C, Roberts H, Taubert KA. Use of nonsteroidal antiinflammatory drugs: an update for clinicians. A scientific statement from the American Heart Association. Circulation 2007;115:1634-42.

27 Antman EM, Hand M, Armstrong PW, et al. 2007 focused update of the ACC/AHA 2004 guidelines for the management of patients with ST-elevation myocardial infarction: a report of the American College of Cardiology/American Heart Association Task Force on Practice Guidelines: developed in collaboration with the Canadian Cardiovascular Society endorsed by the American Academy of Family Physicians: 2007 Writing Group to Review New Evidence and Update the ACC/AHA 2004 Guidelines for the Management of Patients With ST-Elevation Myocardial Infarction, Writing on Behalf of the 2004 Writing Committee. Circulation 2008;117:296-329.

28 Chan FK, Wong VW, Suen BY, et al. Combination of a cyclooxygenase-2 inhibitor and a proton-pump inhibitor for prevention of recurrent ulcer bleeding in patients at very high risk: a double-blind, randomised trial. Lancet 2007;369:1621-6.

29 Scheiman IM. Yeomans ND, Talley NJ, et al. Prevention of ulcers by esomeprazole in at-risk patients using non-selective NSAIDs and COX-2 inhibitors. Am I Gastroenterol 2006;101:701-10

30 Scheiman IM. The use of proton pump inhibitors in treating and preventing NSAID-induced mucosal damage. Arthritis Res Ther 2013;15(suppl 3):S5.

31 Lanas A, Garcia-Rodriguez LA, Arroyo MT, et al. Effect of antisecretory drugs and nitrates on the risk of ulcer bleeding associated with nonsteroidal anti-inflammatory drugs, antiplatelet agents, and anticoagulants. Am I Gastroenterol 2007;102:507-15.

32 Ray WA, Chung CP, Stein CM, et al. Risk of peptic ulcer hospitalization in users of NSAIDs with gastroprotective cotherapy versus coxibs. Gastroenterology 2007;133:790-8

33 Melloni C, Washam JB, Jones WS, et al. Conflicting results between randomized trials and observational studies on the impact of proton pump inhibitors on cardiovascular events when coadministered with dual antiplatelet therapy: systematic review. Circ Cardiovasc Qual Outcomes 2015;8:47-55.

34 Sorensen R, Abildstrom SZ, Weeke P, et al. Duration of clopidogrel treatment and risk of mortality and recurrent myocardial infarction among 11680 patients with myocardial infarction treated with percutaneous coronary intervention: a cohort study. BMC Cardiovas Disord 2010;10:6

35 Schmidt M, Hallas J, Friis S. Potential of prescription registries to capture individual-level use of aspirin and other nonsteroidal anti-inflammatory drugs in Denmark: trends in utilization 1999-2012. Clin Epidemiol 2014;6:155-68.

(c) BMJ Publishing Group Ltd 2015 